\title{
Using DEM to assess the influence of stress and fabric inhomogeneity and anisotropy on susceptibility to suffusion
}

\author{
K. Kawano, C. O’Sullivan \& T. Shire \\ Department of Civil and Environmental Engineering, Imperial College London, UK
}

\begin{abstract}
Underfilled and gap-graded soils are known to be susceptible to suffusion; a form of internal instability in which the finer fraction of a soil is washed out from the coarser matrix under the action of seepage. This phenomenon poses a risk to embankment dams and flood embankments. The processes and mechanisms operate at the particle scale, and insight can be gained via the particulate discrete element method (DEM). Virtual samples can be created using DEM and simulation results can provide information on particle stresses, as well as quantitative information on the fabric of the particulate material. This is important as the amount of stress carried by the finer particles is thought to govern the susceptibility of a given material to suffusion. DEM modelling can also provide information on variation in properties within samples as well as the detailed data needed to quantify the material fabric. DEM models are, however, an idealization of reality and constrained in particular by the number of particles used and sample preparation method. This study examines key issues relating to the development of virtual samples for use in DEM analysis and also examines the proportion of the applied stress that is carried by the finer particles.
\end{abstract}

\section{INTRODUCTION}

\subsection{Background}

Suffusion is a type of internal erosion where the finer grains in a soil are washed out under the action of seepage flow (Moffat et al., 2011; Fannin et al., 2014). Suffusion poses a significant hazard to embankment dams and flood embankments and is considered in recent guidelines for practising engineers: CIRIA et al. (2013) and ICOLD (2015). Materials that are susceptible to suffusion are considered to be internally unstable.

A key contribution is the work of Skempton \& Brogan (1994) who showed that in an internally unstable material significant particle migration can occur at hydraulic gradients of approximately $20 \%$ of the critical hydraulic gradient calculated from Terzaghi's theory $\left(i_{c}\right)$. They attributed this observation to the fact that the finer grains in the sample carry a stress that is significantly lower than the applied stress. Skempton \& Brogan (1994) proposed a stress reduction factor $(\alpha)$ which is the ratio of the stress in the finer fraction $\left(\sigma_{\text {fine }}\right)$ to the overall applied stress $\left(\sigma^{\prime}\right)$ :

$\sigma_{\text {fine }}^{\prime}=\alpha \sigma^{\prime}$
They hypothesised that $\alpha$ can be estimated by comparing the hydraulic gradient at which there is significant particle migration with $i_{\mathrm{c}}$. Shire et al. (2014) used DEM to revisit a sample considered by Skempton \& Brogan (1994) and largely confirmed this hypothesis using particle-scale measurement of stresses.

Shire et al. (2014) directly calculated $\alpha$, rather than inferring it from hydraulic gradients at which particles moved. To calculate $\alpha$ they used the expression for an average stress tensor within a particle given by Potyondy and Cundall (2004):

$\bar{\sigma}_{i j}=\frac{1}{V} \sum_{1}^{N_{p}} \bar{\sigma}_{i j}^{p} V^{p}$

where $\bar{\sigma}_{i j}=$ stress acting throughout volume; $V=$ total volume (particles + voids); $N_{\mathrm{p}}=$ number of particles; $\bar{\sigma}_{i j}^{p}=$ average stress in particle $\mathrm{p}$; and $V^{p}=$ volume of particle p. Using Equation 2, the mean normal stress for fine particles is given as follows:

$\bar{\sigma}_{\text {fine }}=\frac{(1-n)}{\sum_{N_{p}, \text { fine }} V^{p}} \sum_{p=1}^{N_{p, \text { fine }}} \bar{\sigma}_{i}^{p} V^{p}$

where $\bar{\sigma}_{\text {fine }}=$ mean stress acting in the finer particles; $n=$ sample porosity; and $N_{\mathrm{p} \text {,fine }}=$ number of fine particles.

Again, in agreement with hypotheses put forward by Skempton \& Brogan (1994), Shire et al. (2014) 
showed that at a fines contents of $24 \%$ the finer grains do not completely fill the void space between the coarser particles, so they carry little stress, and the material is considered underfilled. At fines contents above $35 \%$, where the finer grains separate the coarser grains, they transmit significant stress, and the material can be considered overfilled. The DEM data highlighted that for transitional fines contents between 24 and 35\% the susceptibility to internal instability is very sensitive to packing density (relative density). Shire et al. (2014) found that for underfilled materials typically $\alpha<0.4$, while typically $\alpha>$ 0.8 for overfilled materials. These conclusions were developed for isotropic stress and fabric and it is not clear whether they are applicable to more general stress states and fabrics.

In contrast to Skempton \& Brogan (1994)'s experiments, where the samples were pluviated under gravity to have a one-dimensional $K_{0}$ stress state, Shire et al. (2014) considered isotropic stress conditions. Chang \& Zhang (2013) showed that the susceptibility to suffusion is dependent on the stressstate. They found that the hydraulic gradients that caused measureable deformation under isotropic stress conditions are much larger than those under triaxial compression or extension. They attributed the difference to the formation and of strong force chains in anisotropic stress states. Ke \& Takahashi (2015) experimentally observed mechanical consequences of suffusion under different effective confining pressures and concluded that the decrease in strength and settlement after suffusion becomes smaller under larger initial effective confining pressure. Again they attributed this to the stability of force chains and the stabilizing contribution of the fine particles that remain after suffusion. Chang \& Zhang (2013) and Ke \& Takahashi (2015) could only propose hypotheses about the mechanics underlying the macro-scale deformation; there is a clear need for additional DEM analyses to examine whether these hypotheses are accurate.

Shire et al. (2014) considered samples in a periodic cell, thus minimising boundary effects. This study extends their contribution to see how sensitive their results are to the way the DEM samples are created. This study also examines the implications of using rigid boundaries for internal stability studies. Rigid wall boundaries are needed in simulations where gravity is applied. Furthermore, not all DEM codes allow use of periodic boundaries and rigid wall boundaries may be needed when coupling DEM with computational fluid dynamics (CFD) programmes to simulate fluid-particle interaction. .

After describing how the DEM simulations were carried out, the paper discusses the homogeneity of the specimens created, prior to discussing anisotropy.

\section{SIMULATION AND ANALYSIS APPROACH}

\subsection{Particle generation}

The DEM simulations discussed here were carried out using the PFC3D software (Itasca Consulting Group (2016)). The samples considered have a gapgraded particle size distribution (PSD) with 35\% fines as illustrated in Figure 1. This particle size distribution was previously considered by Shire (2014) and Shire et al. (2014). Two types of samples were considered: compressed and pluviated. The compressed samples are illustrated in Figure 2; in this case, three numerical samples were prepared to consider size effects on the measured parameters. These samples had differing numbers of particles, the "small" sample had 3,315 particles, the "medium" sample had 15,982 particles and the "large" sample had 31,793 particles. A pluviated sample was also prepared to consider segregation effects as shown in Figure 3. In this case, all 31,793 particles fell under a gravitational body force, and compression was applied subsequently. The equivalent sample considered by Shire et al. (2014) had 50,357 particles.

Kezdi (1979) proposed that internal stability for gap-graded soils can be assessed using the ratio $D^{\prime}{ }_{15} / d^{\prime}{ }_{85}$, where $D^{\prime}{ }_{15}$ stands the coarse fraction diameter for which $15 \%$ of coarse particles are smaller, and $d^{\prime}{ }_{85}$ represents a fine fraction diameter for which $85 \%$ of fine particles are smaller, as shown in Figure 1. In this approach, gap-graded soils are regarded as being internally stable if $D^{\prime}{ }_{15} / d^{\prime}{ }_{85}<$ 4.Referring to Figure 1, in the current study particles more than $0.8 \mathrm{~mm}$ in diameter are considered to be "coarse", while particles less than $0.12 \mathrm{~mm}$ are considered to be "fine". $D{ }_{15} / d$ ' ${ }_{85}$ of the gap-graded soil considered here is 4.6 , meaning that this soil is unstable according to the Kezdi (1979) approach.

Key soil properties and DEM parameters are given in Table 1, which are approximately the same as values for spherical glass beads used by Barreto (2008). The critical time step used in this study was calculated as (Itasca Consulting Group, 2007):

$t_{c r i t}=\sqrt{\frac{m}{k}} \times \beta$

where $t_{\text {crit }}=$ critical timestep; $m=$ mass; $k=$ spring stiffness; and $\beta=0.25$ (this is a safety factor).

Two coefficients of friction $(\mu)$ were used to control the initial void ratio and relative density, so that $\mu=0.1$ gave dense samples and $\mu=0.3$ gave loose samples. 


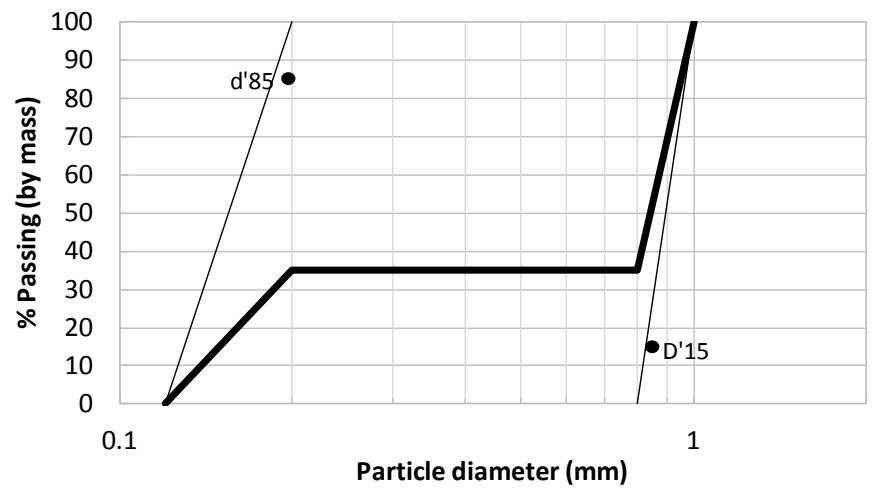

Figure 1. PSD of gap-graded soil with $35 \%$ fines

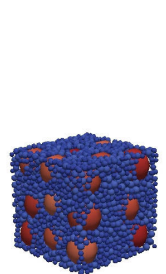

(a) (b) (c)
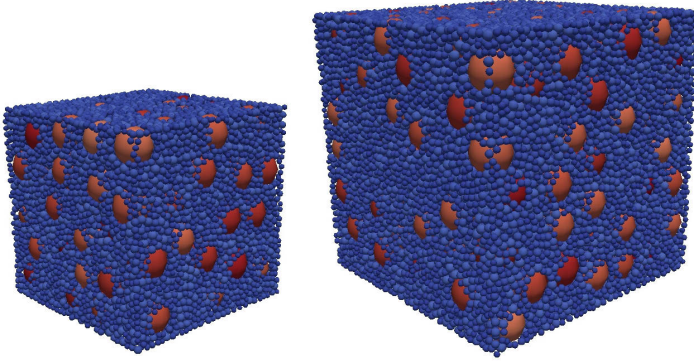

Figure 2. Schematic figures of three compressed numerical samples: (a) Small sample: 3,315 particles; (b) Medium sample: 15,982 particles; (c) Large sample: 31,793 particles

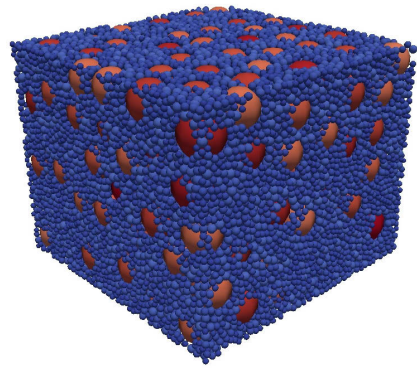

Figure 3. Schematic diagram of pluviated numerical sample: 31,793 particles

Table 1. DEM input parameters

\begin{tabular}{ll}
\hline Parameter & Value \\
\hline Particle density, $\rho$ & $2.670 \mathrm{~kg} / \mathrm{m}^{3}$ \\
Poisson's ratio, $v$ & 0.3 \\
Shear modulus, $G$ & $27.0 \mathrm{GPa}$ \\
$\begin{array}{l}\text { Coefficient of friction, } \mu \\
\quad \text { between particles }\end{array}$ & $0.1,0.3$ \\
$\quad$ between particles and walls & 0.0 \\
Local damping & \\
$\quad$ during compression & 0.1 \\
$\quad$ during applying gravity & 0.05 \\
Gravity, $g$ & $9.80665 \mathrm{~m} / \mathrm{s}^{2}$ \\
Timestep, $\Delta t$ & $2.0 \mathrm{E}-8 \mathrm{~s}$ \\
Contact model & Non-linear Hertz-Mindlin \\
Boundary condition & Rigid wall \\
\hline
\end{tabular}

\subsection{Representative Volume Element (RVE)}

DEM was proposed by Cundall \& Strack (1979) and is now well established as a tool in geomechanics research, e.g. O'Sullivan (2014). DEM simulations are computationally expensive, and the numbers of particles used in geomechanics related DEM simulations rarely exceeds 10,000 according to the work of O'Sullivan (2014). A $1 \mathrm{~cm}^{3}$ cube of uniform sand with median particle diameter $\left(D_{50}\right)$ of 200 microns will contain about 150,000 particles. Thus DEM simulations are using very small numbers of particles; consequently boundary effects and issues around establishing a minimum representative volume element (RVE) are important.

Previous DEM-related research that has investigated boundary effects / sample size effects in terms of bulk properties, such as compressive strength and the critical state line position includes Potyondy \& Cundall (2004); Huang et al. (2014). These studies have been supplemented with observations of boundary effects on porosity, strain and contact force distributions near rigid walls (Chan \& Ng (1986); Marketos \& Bolton (2009); Huang et al. (2014)).

Huang et al. (2014) highlighted the need to check that the overall response is a representative material response when rigid walls are used. The concept of a RVE, which was defined by Nemat-Nasser \& Hori (1999), is useful. A RVE for a material point of a continuum mass is a material volume which is statistically representative of the infinitesimal material neighbourhood of that material point. A RVE must include a very large number of corresponding microconstituents of the RVE, and be statistically representative of the local continuum properties. It must be large enough not to change measured parameters, such as coordination number, porosity, stress and strain. Here, coordination number is defined as the number of contacts per particle; i.e. $z=2 N_{\mathrm{c}} / N_{\mathrm{p}}$ where $z=$ coordination number; $N_{\mathrm{c}}=$ the total number of contacts; $N_{\mathrm{p}}=$ the number of particles. This index is useful for the measurement of packing intensity at the particle scale. In DEM simulations, a specified measurement volume may be created as a measurement sphere within a sample as shown in Figure 4 . When a measurement sphere is big enough, it can be regarded as a RVE. Thus the sample considered itself must be at a minimum large enough to itself be a RVE and if subzones of the sample are used to develop conclusions about material response, these must also be a RVE.

As outlined in Potyondy \& Cundall (2004) particle stresses can be calculated knowing the particle positions and the contact forces. This information can be used to calculate an average stress tensor for the measurement sphere. 


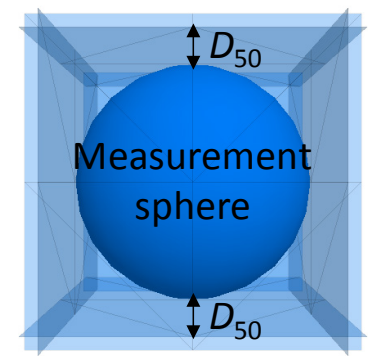

Figure 4. Schematic shape of measurement sphere

\subsection{Isotropic compression and application of gravity}

To create the compressed samples, the particles were initially randomly placed in non-touching positions within a cubic container surrounded by rigid walls, which is called normal method in this study. First, the particle radii were selected to match the gapgraded PSD (Figure 1) using an in-house algorithm, and then all particles were placed by using the PFC3D particle generation algorithm. The drawback of using the PFC3D algorithm is that the resulting samples tend to have an inhomogeneous fabric, so that there is a relatively large concentration of fine particles near the rigid walls. This inhomogeneity arises since this algorithm is designed to place particles in positions which cannot overlap the rigid walls. However, this situation is appropriate for considering real phenomenon as a similar constraint applies close to the boundaries in laboratory experiments.

Each sample was isotropically compressed to a mean stress of $\sigma_{\mathrm{p}}^{\prime}=50 \mathrm{kPa}$ using local damping of 0.1 to minimize dynamic effects. A stress-controlled servo-control algorithm was used during this compression stage. This algorithm applied a controlled strain rate that was gradually reduced as the target isotropic stress of $50 \mathrm{kPa}$ was approached, and the variation in the imposed strain rate with stress is given in Figure 5. This target stress was measured on the walls. After the target stress was reached, and coordination number remained stable for 500,000 steps ( 0.4 seconds in numerical time), the isotropic compression stage was terminated.

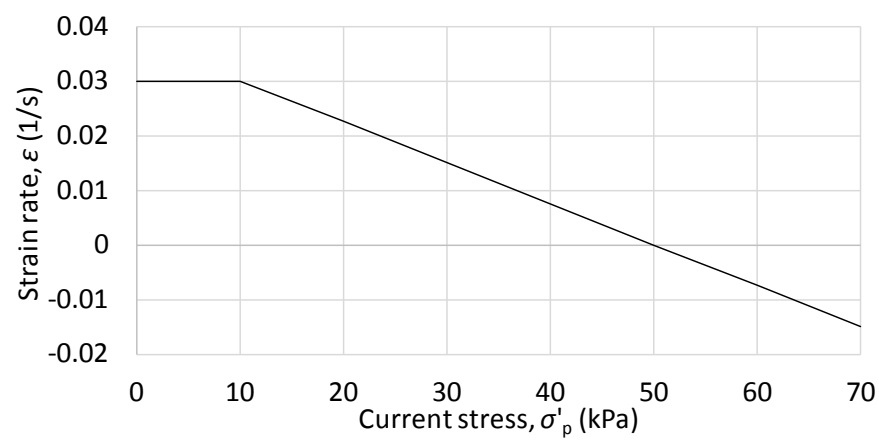

Figure 5. Strain rate applied in accordance with current stress
Once the isotropic stress reached the target stress of $50 \mathrm{kPa}$, gravity was gradually applied to all particles. In order to avoid significant impact forces due to a rapid change in contact forces and prevent segregation, gravity was increased in five stages, i.e. $0.5,1.0,2.0,5.0$ and $9.8 \mathrm{~m} / \mathrm{s}^{2}$. At each stage, once the coordination number stabilised, the next increment of gravity was applied to the sample. After the completion of the application of gravity, the porosity, coordination number and mechanical coordination number were calculated as indicated in Table 2, where the total volume of the sample enclosed by the rigid walls was considered. Mechanical coordination number $\left(z_{\text {mech }}\right)$ was defined by Thornton (2000):

$z_{\text {mech }}=2 \frac{N_{c}-N_{p}^{1}}{N_{p}-\left(N_{p}^{1}-N_{p}^{0}\right)}$

where $N_{\mathrm{p}}^{0}=$ the number of particles with zero contact and $N_{\mathrm{p}}{ }^{1}=$ the number of particles with one contact. Note that contacts between balls and walls are not taken into account in Table 2.

Table 2. Final conditions within the whole sample

\begin{tabular}{llllll}
\hline Sample & $\begin{array}{l}\text { Particles } \\
\text { (Coarse) }\end{array}$ & $\boldsymbol{\mu}$ & $\boldsymbol{n}$ & $\boldsymbol{z}$ & $\boldsymbol{z}_{\text {mech }}$ \\
\hline Small-loose & $3,315(25)$ & 0.3 & 0.298 & 4.49 & 4.52 \\
Medium-loose & $15,982(125)$ & 0.3 & 0.292 & 4.65 & 4.66 \\
Large-loose & $31,793(250)$ & 0.3 & 0.278 & 4.69 & 4.70 \\
Small-dense & $3,315(25)$ & 0.1 & 0.275 & 4.82 & 4.84 \\
Middle-dense & $15,982(125)$ & 0.1 & 0.261 & 5.09 & 5.10 \\
Large-dense & $31,793(250)$ & 0.1 & 0.271 & 5.17 & 5.18 \\
Pluviated & $31,793(250)$ & 0.3 & 0.280 & 4.69 & 4.70 \\
\hline
\end{tabular}

$\mu$ : Coefficient of friction, $n$ : porosity calculated from whole sample, $z$ : coordination number, $z_{\text {mech }}$ : mechanical coordination number

In Figure 6, it is shown that the increase of coordination number affects the decrease of porosity. There is a clear relation between the porosity and coordination number.

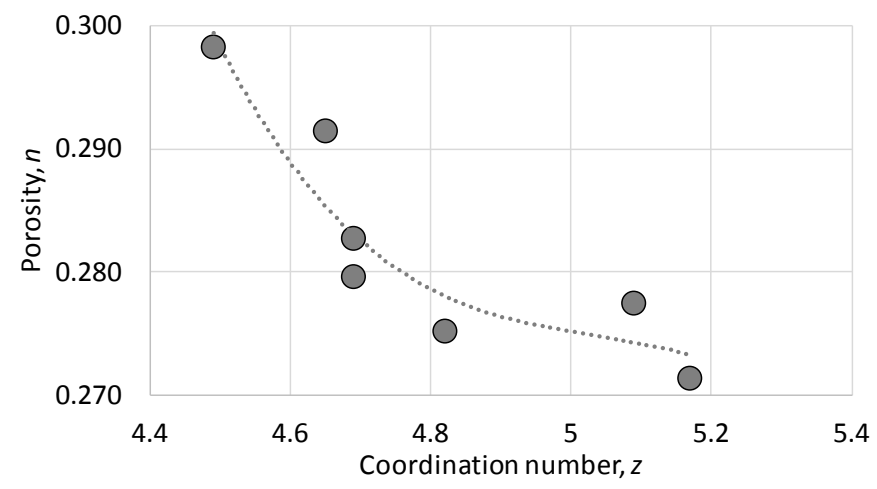

Figure 6. Relation between porosity and coordination number

A pluviated sample with $\mu=0.3$ was also created so as to investigate whether generating a sample by pluviation would induce segregation. To create the pluviated sample, gravity was applied in five stages 
$\left(0.5,1.0,2.0,5.0\right.$ and $\left.9.8 \mathrm{~m} / \mathrm{s}^{2}\right)$. In each step, particles naturally fell down until a steady state was reached when coordination number was stable situation for 500,000 steps $(0.4$ seconds in numerical time). Then, isotropic compression was applied up to the target stress of $50 \mathrm{kPa}$. The final conditions obtained from the measurement sphere are compared in Table 4. The data for "Gap Med 35" included on Table 4, come from the results of Shire et al. (2014) who used periodic boundaries and the same grading.

The values for $n$ and $z$ were calculated by considering the measurement sphere to give $n_{\text {meassphere }}$ and $z_{\text {meas,sphere. }}$ For each sample, measurement sphere was located so that it did not intercept the boundaries and centred at the centre of the sample. For the compressed samples, different measurement sphere radii were considered, extending to 20,100 and $200 \%$ of a median diameter $\left(D_{50}\right)$ away from the boundaries so that the effect of the size of measurement spheres can be compared. In this study, the $D_{50}$ for the entire grading was used which was $0.8 \mathrm{~mm}$. As Table 3 below shows, for these radii values, the measurement sphere size measurably influence the porosities and coordination numbers for the smaller sample; the data for the larger sample show less variation with measurement sphere size. A measurement sphere which extended to be $100 \%$ of $D_{50}$ from the walls was used in the remaining calculations (Figure 4).

Table 3. Effect of measurement sphere size

\begin{tabular}{llll}
\hline Sample & $\begin{array}{l}\text { Distance from } \\
\text { walls }\end{array}$ & $\boldsymbol{n}_{\text {meas,sphere }}$ & $\boldsymbol{z}_{\text {meas,sphere }}$ \\
\hline Small-loose & & & \\
& 20\% of $D_{50}$ & 0.233 & 5.12 \\
& $100 \%$ of $D_{50}$ & 0.233 & 5.12 \\
Large-dense & 200\% of $D_{50}$ & 0.238 & 4.95 \\
& & & \\
& 20\% of $D_{50}$ & 0.238 & 5.51 \\
& $100 \%$ of $D_{50}$ & 0.237 & 5.50 \\
& $200 \%$ of $D_{50}$ & 0.239 & 5.45 \\
\hline
\end{tabular}

$n_{\text {meas,sphere }}$ : porosity from measurement sphere, $z_{\text {meas,sphere }}$ coordination number from measurement sphere

Referring to the measurement sphere data Table 4 , as expected, the denser samples have lower porosities and higher coordination numbers than the looser samples. In terms of the porosity, the results are in good agreement with Shire et al. (2014); however, Shire et al. (2014) reported lower coordination numbers. The absence of gravity in these earlier simulations allows particles to exist in a state of suspension in voids with no contacts, resulting in lower coordination numbers in comparison with the simulation data presented here. The porosity and coordination number data for the pluviated sample are similar to the large loose compressed sample. Here, $\mu=0.3$ gives a loose packing.
Table 4. Final conditions within measurement sphere

\begin{tabular}{|c|c|c|c|c|}
\hline Sample & $\begin{array}{l}\text { Particles } \\
\text { (Coarse) }\end{array}$ & $\mu$ & $\boldsymbol{n}_{\text {meas,sphere }}$ & $z_{\text {meas,sphere }}$ \\
\hline \multicolumn{5}{|l|}{ Normal method } \\
\hline Small-loose & $3,315(25)$ & 0.3 & 0.233 & 5.12 \\
\hline Medium-loose & $15,982(125)$ & 0.3 & 0.243 & 4.92 \\
\hline Large-loose & $31,793(250)$ & 0.3 & 0.244 & 4.90 \\
\hline Small-dense & $3,315(25)$ & 0.1 & 0.223 & 5.63 \\
\hline Middle-dense & $15,982(125)$ & 0.1 & 0.233 & 5.49 \\
\hline Large-dense & $31,793(250)$ & 0.1 & 0.237 & 5.50 \\
\hline \multicolumn{5}{|c|}{ Pluviated method } \\
\hline Large-dense & $31,793(250)$ & 0.3 & 0.252 & 4.91 \\
\hline \multicolumn{5}{|c|}{ Shire et al. (2014) } \\
\hline Gap Med 35 & $50,357(500)$ & 0.3 & 0.245 & 3.08 \\
\hline Gap Med 35 & $50,357(500)$ & 0.1 & 0.241 & 4.62 \\
\hline
\end{tabular}

\section{INHOMOGENEITY EVALUATION}

\subsection{Layer analysis}

In order to quantify inhomogeneity within the samples, each sample was divided into an integer number of layers to give a layer depth of approximately $0.5 \mathrm{~mm}$, following the approach that was originally developed for cylindrical rigid-wall samples by Huang et al. (2014). The small samples were divided into 6 layers, the medium samples 9 layers and the large samples 12 layers. This layering technique was applied in the $\mathrm{x}, \mathrm{y}$, and $\mathrm{z}$ directions. The volume of particles within each layer was accurately calculated by taking the intersection volume into account by determining the volume of the spherical cap, which is created when a sphere intersects a layer boundary. Figure 7 displays the spherical cap, and the corresponding volume is given in Equation 6.

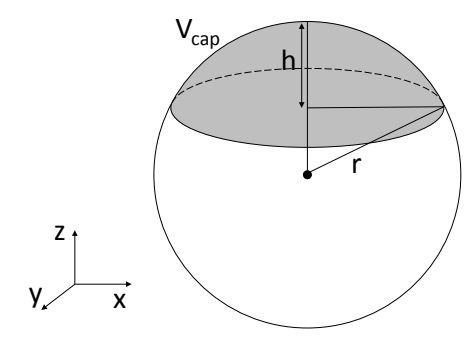

Figure 7. Schematic area of spherical cap

$V_{c a p}=\frac{\pi h^{2}(3 r-h)}{3}$

where $V_{\text {cap }}=$ spherical cap (hatching area of Figure 7 ); $h=$ height of spherical cap; and $r=$ radius.

Representative distributions of porosity in all three coordinate directions (normalized by mean porosity excluding values in both end layers) are shown in Figure 8 and Figure 9, and the detailed measurements for all samples considered are provided in Table 5 considering the vertical $(\mathrm{z})$ direction. It is clear that in all cases the porosity close to the top and bot- 
tom boundaries is higher than the average porosity in agreement with Chan \& Ng (1986), Marketos \& Bolton (2009) and Huang et al. (2014). There is also significant variation within the sample, and this variation is more noticeable for the smaller samples, reflected in the higher standard deviation associated with these samples (Table 5). The greater variation in measured porosity in the layers in the smaller sample can simply be attributed to the fact that each layer is significantly smaller than a RVE and so the position and size of one particle within that layer can have a measurable influence on the porosity for that layer. Although the average porosity in Table 5 is not very different from the porosity within the measurement sphere in Table 4, especially for the large samples, the tolerance between these results is still approximately $9 \%$. Considering the measurement sphere is useful for thinking of homogeneity, and laboratory experiments need to be taken into account the inhomogeneity.

The PSDs in each layer were compared to understand the inhomogeneity. Referring to the smallloose sample (Figure 10), large-dense sample (Figure 11) and pluviated sample (Figure 12), while there is some evidence of segregation in the smallloose sample, the PSDs of the large-dense and pluviated sample are very similar to each other, excluding the top and bottom layers, and so this sample can be regarded as being homogeneous (no segregation).

As Figure 11 illustrates, the large-dense sample shows that more fine particles tend to be placed in bottom and top layers due to the walls. In particular, the bottom layer of the pluviated sample (Figure 12) is inclined to have more fines than that of largedense sample. More interestingly, it is seen that No. 2 (second layer from the top layer) has slightly more coarse particles because of gravity applied. Note also that the PSDs of layers No. 1 (Top layer) and No. 12 (Bottom layer) are broadly similar in this case.

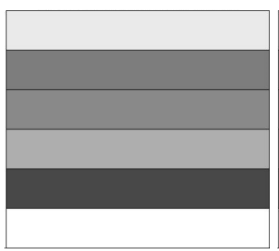

(a)

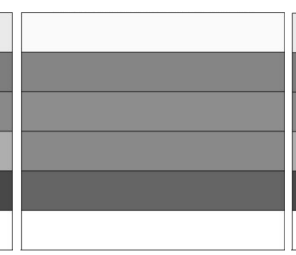

(b)

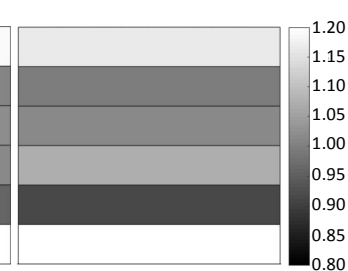

(c)
Figure 8. Normalised distribution of porosities with small-loose sample: (a) in x-direction; (b) in y-direction; (c) in z-direction

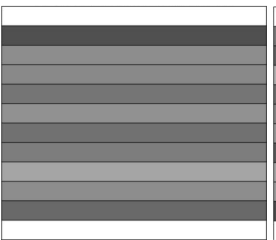

(a)

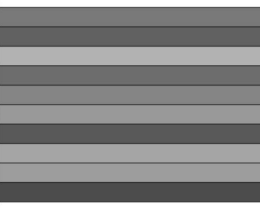

(b)

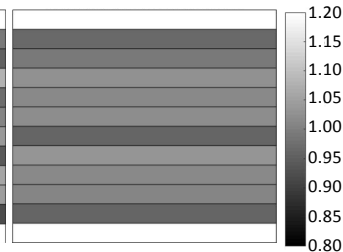

(c)
Figure 9. Normalised distribution of porosities with large-dense sample: (a) in $\mathrm{x}$-direction; (b) in y-direction; (c) in z-direction

Table 5. Porosity distribution of each layer in $\mathrm{z}$ direction

\begin{tabular}{|c|c|c|c|c|c|c|c|}
\hline \multirow{2}{*}{$\begin{array}{l}\text { Laye } \\
\text { No. }\end{array}$} & \multicolumn{2}{|c|}{ Small } & \multicolumn{2}{|c|}{ Medium } & \multicolumn{2}{|c|}{ Large } & \multirow{2}{*}{$\begin{array}{l}\text { Pluviatec } \\
\text { Loose }\end{array}$} \\
\hline & Loose & Dense & Loose & Dense & Loose & Dense & \\
\hline \multicolumn{8}{|c|}{ Top layer } \\
\hline 1 & - & - & - & - & 0.343 & 0.341 & 0.331 \\
\hline 2 & - & - & - & - & 0.253 & 0.240 & 0.241 \\
\hline 3 & - & - & - & - & 0.269 & 0.264 & 0.281 \\
\hline 4 & - & - & 0.321 & 0.318 & 0.265 & 0.262 & 0.260 \\
\hline ) & - & - & 0.269 & 0.252 & 0.264 & 0.255 & 0.272 \\
\hline 6 & - & - & 0.270 & 0.266 & 0.267 & 0.265 & 0.270 \\
\hline 7 & 0.325 & 0.325 & 0.284 & & 0. & & 0.264 \\
\hline 8 & 0.278 & 0.268 & 0.259 & 0.254 & 0.268 & 0.257 & 0.268 \\
\hline 9 & 0.283 & 0.278 & 0.281 & 0.274 & 0.277 & 0.274 & 0.278 \\
\hline 10 & 0.299 & & 0.265 & & & & 0.292 \\
\hline 11 & 0.254 & 0.246 & 0.267 & 0.256 & $0.25 \mathrm{c}$ & 0.249 & 0.252 \\
\hline 12 & 0.351 & 0.346 & 0.329 & 0.328 & 0.337 & 0.334 & 0.348 \\
\hline \multicolumn{8}{|c|}{ Bottom layer } \\
\hline & 0.279 & 0.270 & 0.271 & 0.262 & 0.26 & 0.258 & 0.268 \\
\hline & 0.019 & 0.017 & 0.009 & 0.009 & 0.007 & 0.010 & 0.015 \\
\hline
\end{tabular}

$\mu$ : average without both end layers, $\sigma$ : standard deviation without both end layers

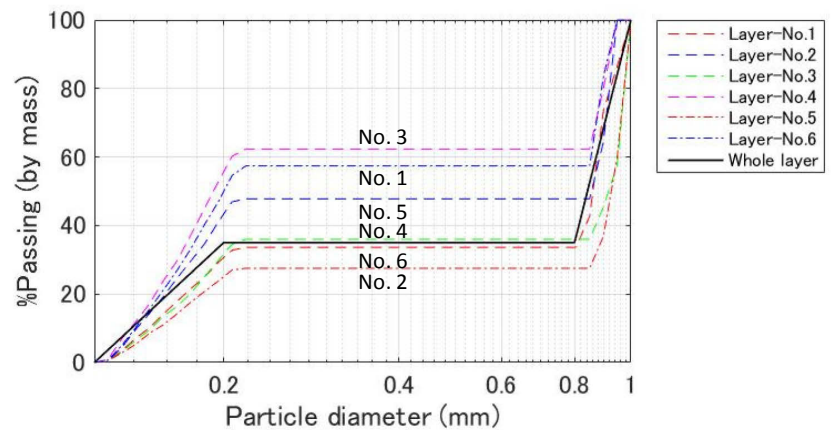

Figure 10. PSDs in each layer of small-loose sample

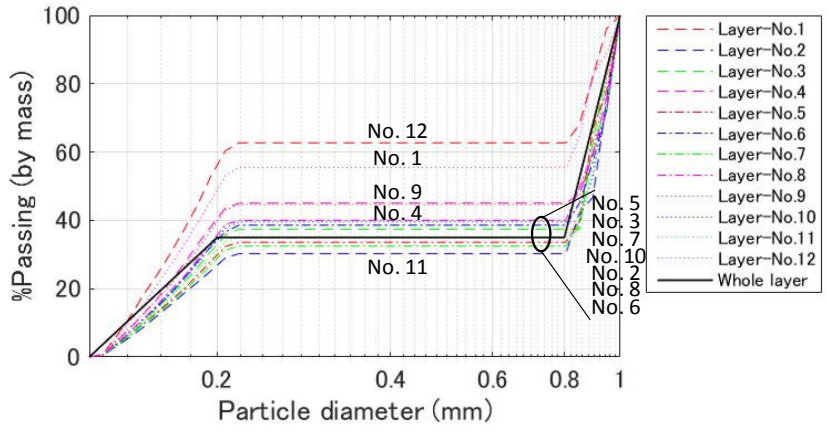

Figure 11. PSDs in each layer of large-dense sample 


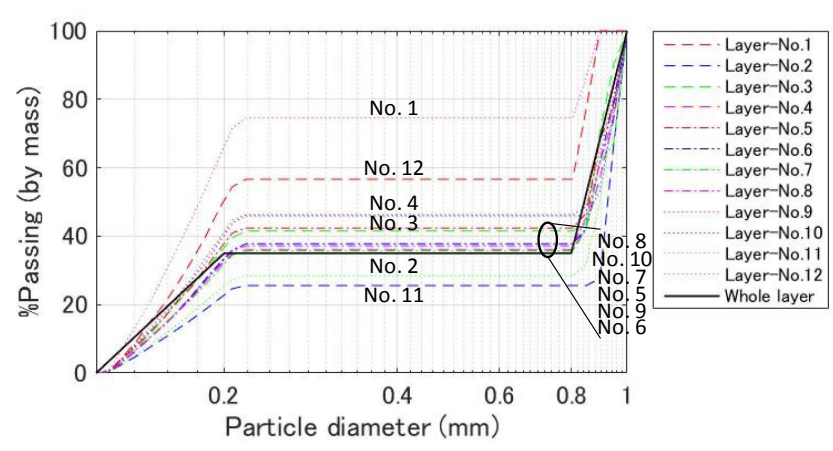

Figure 12. PSDs in each layer of pluviated sample

\subsection{Comparison of measurement sphere with overall data}

One potential way to remove boundary effects on the calculated $\alpha$ values is to consider only particles within the central measurement sphere. To isolate this sub-set of particles, the particles that intersected the measurement sphere were identified, as shown in Figure 13. Then, as indicated in Figure 14, the volume of a sphere-sphere intersection was calculated using Equation 7. Thus the partial volumes of the intersected particles that were considered are as illustrated in Figure 15.

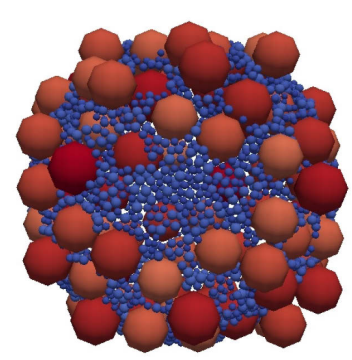

(a)

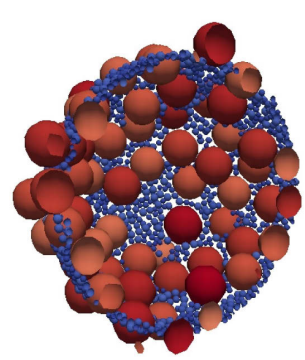

(b)
Figure 13. Particles on the intersection: (a) all particles; (b) cross-section view along the centre plane

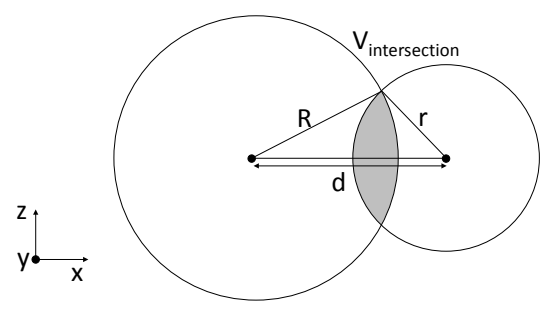

Figure 14. Schematic area of sphere-sphere intersection

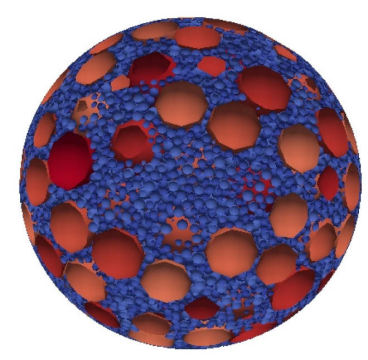

Figure 15. Particles including intersected particles within measurement sphere
$V_{\text {inter section }}=\frac{\pi(R+r-d)^{2}\left(d^{2}+2 d r-3 r^{2}+2 d R+6 r R-3 R^{2}\right)}{12 d}$

where $V_{\text {intersection }}=$ sphere-sphere intersection (hatching area of Figure 14); $R=$ radius of one particle; $r=$ radius of the other particle; and $d=$ distance between centres of two spheres.

In Figure 16 and Figure 17, the distribution of mean particle stress is visualised on the three samples on an XZ-plane through the samples. Particles shaded in "black" transfer a significant amount of stress, whereas the "white" particles transfer substantially less stress. For the loose samples in Figure 16 , stress is preferentially transmitted by the coarse particles, when the centre of the figure is considered. As density increases, fines carry more stress as shown by comparing Figure 16 and Figure 17, where the loose samples are considered, there is a higher proportion of low stress "white" particles in the centre of the samples than for the dense samples in Figure 17. The increase of $\alpha$ values with sample density (Table 6 and Table 7) confirms the observations of Shire et al. (2014) that this type of transitional fine content is sensitive to relative density. The heterogeneity in the smaller samples is very evident in Figure 16 and Figure 17. In particular, the force chain is formed by only a few coarse particles. The small numbers of highly stressed particles in this small sample in Figure 16(a) highlights the fact that this sample is too small to be considered a RVE.

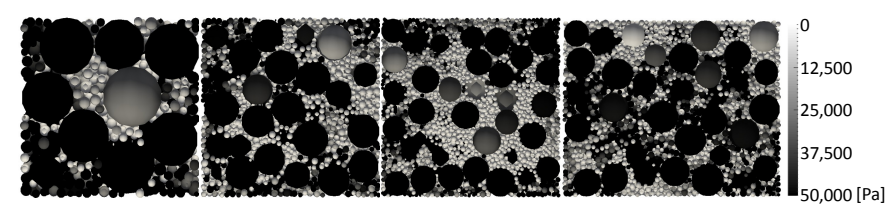

(a)

(b)

(c)

(d)

Figure 16. Distribution of mean stress in XZ plane with loose samples: (a) small; (b) medium; (c) large; (d) pluviated

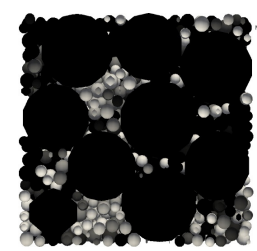

(a)

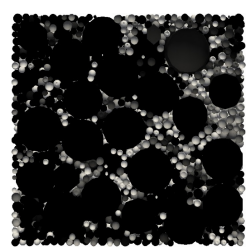

(b)

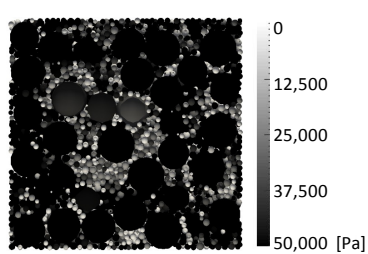

(c)
Figure 17. Distribution of mean stress in XZ plane with dense samples: (a) small; (b) medium; (c) large

Table 6 and Table 7 give the $\alpha$ values obtained from the whole sample and the measurement sphere, respectively. There are measureable differences when the $\alpha$ values in these two tables are compared. The values listed in Table 6 are significantly higher than the values listed in Table 7. The ratio of $\alpha$ calculated from the whole sample to $\alpha$ obtained from the measurement sphere is also shown in Table 8 . 
These data show the concentration of fines close to the boundary lead to an unrealistic increase in $\alpha$ values. Furthermore in laboratory tests observations of particle migration close to the boundaries will not be representative of the material response.

The data presented in Table 6 and Table 7 indicate that the $\alpha$ values for the loose samples are smaller than those of the dense samples. Considering the measurement sphere data in Table 7, for the loose samples, the $\alpha$ value is roughly between 0.34 and 0.51 . The $\alpha$ value of the dense samples are between 0.69 and 0.84 . The $\alpha$ value increases as the number of particles increases.

As was the case for the porosity data in Table 4, the $\alpha$ values noted by Shire et al. (2014) are included on Table 7. The results show that for large samples, provided the boundary data are excluded, representative data can be obtained.

Table 6. Stress reduction factor calculated from throughout the whole sample including stress in both ends of layers

\begin{tabular}{|c|c|c|c|c|c|c|}
\hline \multirow[b]{2}{*}{ Direction } & \multicolumn{2}{|c|}{ Small } & \multicolumn{2}{|c|}{ Middle } & \multicolumn{2}{|c|}{ Large } \\
\hline & Loose & Dense & Loose & Dense & Loose & Dense \\
\hline$\alpha_{\text {whole }}$ & & & & & & \\
\hline$\alpha, \mathrm{p}^{\prime}$ & 0.791 & 0.918 & 0.687 & 0.976 & 0.641 & 0.991 \\
\hline$\alpha, \sigma_{\mathrm{x}}$ & 0.750 & 0.892 & 0.681 & 0.962 & 0.626 & 0.987 \\
\hline$\alpha, \sigma_{\mathrm{y}}$ & 0.845 & 0.951 & 0.695 & 0.973 & 0.635 & 0.994 \\
\hline$\alpha, \sigma_{\mathrm{z}}$ & 0.779 & 0.912 & 0.685 & 0.994 & 0.662 & 0.991 \\
\hline
\end{tabular}

$\alpha, \mathrm{p}^{\prime}: \alpha$ calculated from mean stress, $\alpha, \sigma_{\mathrm{x}}: \alpha$ relating to $\mathrm{x}$ direction, $\alpha, \sigma_{\mathrm{y}}: \alpha$ relating to $\mathrm{y}$ direction, $\alpha, \sigma_{\mathrm{z}}: \alpha$ relating to $\mathrm{z}$ direction

Table 7. Stress reduction factor calculated from particles within measurement sphere

\begin{tabular}{|c|c|c|c|c|c|c|}
\hline \multirow[b]{2}{*}{ Direction } & \multicolumn{2}{|c|}{ Small } & \multicolumn{2}{|c|}{ Middle } & \multicolumn{2}{|c|}{ Large } \\
\hline & Loose & Dense & Loose & Dense & Loose & $\overline{\text { Dense }}$ \\
\hline \multicolumn{7}{|l|}{$\alpha_{\text {meas,sphere }}$} \\
\hline$\alpha, \mathrm{p}^{\prime}$ & 0.506 & 0.694 & 0.430 & 0.830 & 0.341 & 0.836 \\
\hline$\alpha, \sigma_{\mathrm{x}}$ & 0.421 & 0.652 & 0.428 & 0.814 & 0.335 & 0.823 \\
\hline$\alpha, \sigma_{\mathrm{y}}$ & 0.616 & 0.757 & 0.437 & 0.839 & 0.327 & 0.850 \\
\hline \multirow[t]{2}{*}{$\alpha, \sigma_{\mathrm{z}}$} & 0.491 & 0.678 & 0.426 & 0.837 & 0.361 & 0.835 \\
\hline & & & \multicolumn{2}{|c|}{ Gap Med 35} & \multicolumn{2}{|c|}{ Pluviated } \\
\hline$\alpha, \mathrm{p}^{\prime}$ & - & - & 0.34 & 0.86 & 0.552 & - \\
\hline$\alpha, \sigma_{\mathrm{x}}$ & - & - & - & - & 0.509 & - \\
\hline$\alpha, \sigma_{\mathrm{y}}$ & - & - & - & - & 0.606 & - \\
\hline$\alpha, \sigma_{\mathrm{z}}$ & - & - & - & - & 0.548 & - \\
\hline
\end{tabular}

Table 8. Ratio of stress reduction from measurement sphere to that from the whole sample

\begin{tabular}{|c|c|c|c|c|c|c|}
\hline \multirow[b]{2}{*}{ Direction } & \multicolumn{2}{|c|}{ Small } & \multicolumn{2}{|c|}{ Middle } & \multicolumn{2}{|c|}{ Large } \\
\hline & Loose & $\overline{\text { Dense }}$ & Loose & Dense & Loose & Dense \\
\hline \multicolumn{7}{|c|}{$\alpha_{\text {meas,sphere }} / \alpha_{\text {whole }}$} \\
\hline$\alpha, \mathrm{p}^{\prime}$ & 0.64 & 0.76 & 0.63 & 0.85 & 0.53 & 0.84 \\
\hline$\alpha, \sigma_{\mathrm{x}}$ & 0.56 & 0.73 & 0.63 & 0.85 & 0.54 & 0.83 \\
\hline$\alpha, \sigma_{\mathrm{y}}$ & 0.73 & 0.80 & 0.63 & 0.86 & 0.51 & 0.86 \\
\hline$\alpha, \sigma_{\mathrm{z}}$ & 0.63 & 0.74 & 0.62 & 0.84 & 0.55 & 0.84 \\
\hline
\end{tabular}

\section{ANISOTROPY}

\subsection{Stress-induced anisotropy}

For the samples that were initially isotropically consolidated, prior to applying gravitational loading, there is a small stress anisotropy owing to the weight of particles in the vertical (z) direction. The $\alpha$ values were calculated so as to get a feel as to whether such an anisotropy might influence susceptibility to internal erosion. The difference in stress was, however, small. The isotropic stress applied was $50 \mathrm{kPa}$. The additional stress in the vertical $(\mathrm{z})$ direction produced by applying gravity to 31,793 particles was approximately $116 \mathrm{~Pa}$. This stress is not capable of contributing the anisotropy. However, particle rearrangement caused by gravity could still include anisotropy.

As for the $\alpha$ values as shown in Table 7, discrepancy between directions becomes narrow with the large number of particles and high density. Focusing on the large samples, the difference of $\alpha$ values in $\mathrm{x}$ and y direction to $\alpha$ values in $\mathrm{z}$ direction is at most $3 \%$ in the dense sample, whereas the difference is $10 \%$ in the loose sample. The effect of particle configuration predominantly affects the $\alpha$ values difference when the applied stress was distributed. Considering the heterogeneity in the small samples, segregation and size deficiency of the measurement sphere, which is not large enough as the RVE, the $\alpha$ values are not taken into account here. The medium and large samples probably have a RVE according to the data. The stress-induced anisotropy can be measurably recognised in the samples with lower densities and pluviated sample.

\subsection{Fabric anisotropy}

Oda et al. (1985) showed that fabric can be quantified by using orientation data at particle scale: contact normal, particle, void and branch vector. Here, fabric anisotropy was quantified by means of unit contact vectors normal as well as Oda et al. (1985). The direction of contacts between particles is visualised from Figure 18 and Figure 19. Here, each rose histogram bin length represents the number of all contacts orientated within that bin's angular increment. 36 bins were considered to give $10^{\circ}$ angular increments. The colour gradation stands for the magnitude of average contact force with a specific direction. Satake (1982) defined fabric tensor for quantifying the fabric anisotropy as follows:

$$
\Phi_{i j}=\frac{1}{N} \sum_{k=1}^{N} n_{i}^{k} n_{j}^{k}
$$

where $N=$ total number of vectors; and $\theta=$ direction of the fabric anisotropy or principal fabric. This fabric tensor has been used in a number of DEM stud- 
ies; for example, Thornton (2000) and Shire et al. (2012). Rothenburg \& Bathurst (1989) proposed an equation including only two parameters where an ellipse is fitted by means of Fourier series. The Satake (1982) equation and Rothenburg \& Bathurst (1989) equation are shown respectively;

$$
E(\theta)=\frac{1}{2 \pi}\left\{1+a \cos 2\left(\theta-\theta_{a}\right)\right\}
$$

where $a=$ magnitude of fabric anisotropy; and $=k$ th unit orientation vector.

The length of the histograms differs as there are fewer contacts between the particles in the loose samples (Figure 18) than in the dense samples (Figure 19) and pluviated sample (Figure 20). This is related to the coordination number data shown in Table 2. For small number of particles such as Figure 18 , the direction of the contacts slightly varies as the fabric tensor indicates. As the number of particles goes bigger and bigger, this variability becomes small and stable. These figures imply that there is almost no fabric anisotropy due to gravity under 50 $\mathrm{kPa}$. The isotropic compression stress of $50 \mathrm{kPa}$ is relatively big in comparison with the impact of the gravity.

Besides, $\alpha, \sigma_{\mathrm{y}}$ tends to have relatively higher values as shown in Table 6 and Table 7. This may as well be attributed to the way of applying isotropic compression, i.e. the target stress should be controlled by measuring stress within a measurement sphere instead of walls. Further investigation is required.

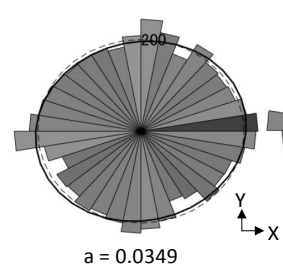

(a)

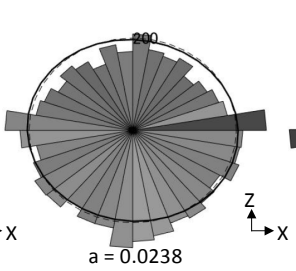

(b)

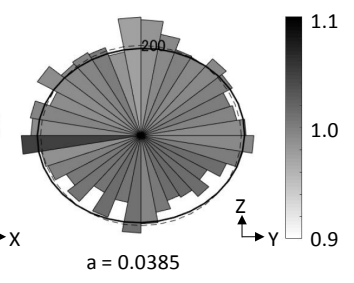

(c)
Figure 18. Rose histograms of fabric anisotropy with smallloose sample: (a) in XY-plane; (b) in XZ-plane; (c) in YZ-plane

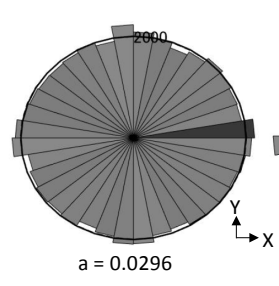

(a)

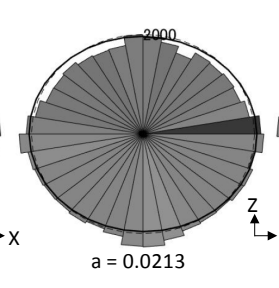

(b)

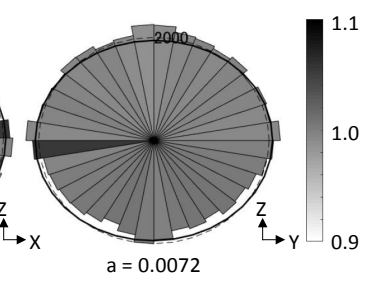

(c)
Figure 19. Rose histograms of fabric anisotropy with largedense sample: (a) in XY-plane; (b) in XZ-plane; (c) in YZplane

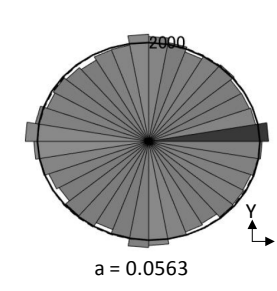

(a) (b)

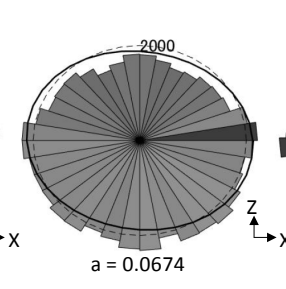

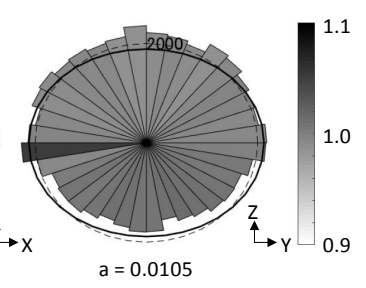

(c)
Figure 20. Rose histograms of fabric anisotropy with pluviated sample: (a) in XY-plane; (b) in XZ-plane; (c) in YZ-plane

\section{CONCLUSIONS}

This contribution has explored how best to run DEM simulations to improve understanding of internal instability. The work builds on the contribution by Shire et al. (2014). Shire et al. (2014) considered periodic boundaries and a gravity-free environment. In this contribution, samples were bounded by rigid walls, and gravity was applied in a controlled manner. The number of particles in the systems varied, and two different interparticle friction values were used to control the relative densities, so that loose and dense samples were considered.

The use of rigid wall boundaries induced significant boundary effects on the samples. The porosities close to the rigid walls were quite high in comparison with those in the centre of the samples. There was also a stress concentration along the rigid walls. The differences between the $\alpha$ values calculated from the central measurement sphere and those obtained from the whole sample emphasized this fact.

There was little particle segregation in the centre of samples when gravity applied, this is most likely a consequence of the way the samples were prepared, e.g. the strategy of the applying gravity in five stages.

Specific recommendations are:

- 3D simulations with more than 30,000 particles are required to obtain representative results for this grading.

- A measurement sphere must be used to interpret DEM data when rigid walls are used in order to avoid the effect of the concentrated stress in the vicinity of the walls and the sample inhomogeneity.

The anisotropy was described in terms of the stress and the fabric. According to the $\alpha$ values in $\mathrm{x}$, $\mathrm{y}$ and $\mathrm{z}$ direction, little anisotropy was observed when there are enough particles, i.e. more than 30,000 particles, whereas the stress-induced anisotropy is seen in the samples with the low density and pluviated sample. Particularly, the $\alpha$ values of the pluviated sample are higher than those of the largeloose sample. In order to avoid this difference, the target stress should be controlled by measuring stress within a measurement sphere. Under the isotropic stress of $50 \mathrm{kPa}$, gravity did not play an important 
role in terms of the anisotropy. The fabric anisotropy can be negligible when the effect of the gravity is relatively smaller than the compression stress.

The results presented here focus on the case only without water. The authors are currently using coupled DEM-CFD analysis to explore how erosion of fine particles with various stress reduction factors initiates.

\section{ACKNOWLEDGMENTS}

This study has been financially supported by Kajima Corporation in Japan.

\section{REFERENCES}

Barreto, D., (2008). Numerical and experimental investigation into the behavior of granular materials under generalized stress states. Ph. D. thesis, Imperial College London, London.

Chan, S.K. \& Ng, K.M., (1986). Geometrical Characteristics of a Computer-generated Three-dimensional Packed Column of Equal and Unequal Sized Sphereswith Special Reference to Wall Effects. Chemical Engineering Communications, 48(4-6), pp.215-236.

Chang, D.S. \& Zhang, L.M., (2013). Critical Hydraulic Gradients of Internal Erosion under Complex Stress States. Journal of Geotechnical and Geoenvironmental Engineering, 139(9), pp.1454-1467.

Chang, D.S., Zhang, L. \& Xu, T.H., (2012). Laboratory Investigation of Initiation and Development of Internal Erosion in Soils under Complex Stress States Key words. , pp.895-902.

CIRIA, French Ministry of Ecology \& USACE, (2013). The International Levee Handbook,

Cundall, P.A. \& Strack, O.D.L., (1979). A discrete numerical model for granular assemblies. Géotechnique, 29(1), pp.47-65.

Ergun, S., (1979). Chemical Engineering Progres s.

Fannin, R.J. et al., (2014). On the distinct phenomena of suffusion and suffosion. Geotechnique Letters, 5, pp.129-130.

Huang, X. et al., (2014). Effect of sample size on the response of DEM samples with a realistic grading. Particuology, 15, pp.107-115.

ICOLD, (2015). ICOLD Bulletin 164: Internal erosion of existing dams, levees, and dikes, and their foundations. Volume 1, 1.

Itasca Consulting Group, I., (2016). PFC3D: Particle flow code in three dimensions.

Itasca Consulting Group, I., (2007). PFC3D Version 4.0 User Manual, Minneapolis.

Ke, L. \& Takahashi, A., (2015). Drained Monotonic Responses of Suffusional Cohesionless Soils. Journal of Geotechnical and Geoenvironmental Engineering, 141(8), pp.1-8.
Marketos, G. \& Bolton, M.D., (2009). Flat boundaries and their effect on sand testing. International Journal for Numerical and Analytical Methods in Geomechanics, pp.821-837.

Moffat, R., Fannin, R.J. \& Garner, S.J., (2011). Spatial and temporal progression of internal erosion in cohesionless soil. Canadian Geotechnical Journal, 48(3), pp.399-412.

Nemat-Nasser, S. \& Hori, M., (1999). Micromechanics: Overall Properties of Heterogeneous Materials 2nd edition, North Holland.

Oda, M., Nemat-Nasser, S. \& Konishi, J., (1985). StressInduced Anisotropy In Granular Masses. Soils and Foundations, 25(3), pp.85-97.

O'Sullivan, C., (2014). Advancing geomechanics using DEM. The International Symposium on Geomechanics from Micro to Macro (IS-Cambridge 2014), pp.21-32.

Potyondy, D.O. \& Cundall, P.A., (2004). A bondedparticle model for rock. International Journal of Rock Mechanics and Mining Sciences, 41(8 SPEC.ISS.), pp.1329-1364.

Rothenburg, L. \& Bathurst, R., (1989). Analytical study of induced anisotropy granular materials. Géotechnique, 39(4), pp.601-614.

Satake, M., (1982). Fabric tensor in granular materials. Proceedings of the IUTAM Conference on Deformation and Failure of Granular Materials. pp. 63-68.

Shire, T. et al., (2012). Quantifying stress-induced anisotropy using inter-void constrictions. Géotechnique, 63(1), pp.85-91.

Shire, T., (2014). Micro-scale Modelling of Granular Filters. Ph. D. thesis, Imperial College London, London.

Shire, T., Hanley, K.J. \& Fannin, R.J., (2014). Fabric and Effective Stress Distribution in Internally Unstable Soils. Journal of Geotechnical and Geoenvironmental Engineering, pp.1-11.

Skempton, a. W. \& Brogan, J.M., (1994). Discussion: Experiments on piping in sandy gravels. Géotechnique, 45(3), pp.565-567.

Thornton, C., (2000). Numerical simulations of deviatoric shear deformation of granular media. Géotechnique, 50(1), pp.43-53. 